\title{
Exploiting Optimal Throughput of Adaptive Relaying Based Wireless Powered Systems under I mpacts of Co-channel I nterference
}

\author{
Thanh-Luan Nguyen ${ }^{1}$, Dinh-Thuan Do ${ }^{2}$ \\ ${ }^{1}$ Department of Communications Engineering, Bach Khoa University, \\ Ho Chi Minh City, Vietnam \\ [e-mail: luannguyen.cce@gmail.com] \\ ${ }^{2}$ Wireless Communications Research Group, Faculty of Electrical and Electronics Engineering, \\ Ton Duc Thang University \\ Ho Chi Minh City, Vietnam \\ [e-mail: luannguyen.cce@gmail.com; dodinhthuan@tdt.edu.vn] \\ *Corresponding author: Dinh-Thuan Do
}

Received August 29, 2017; revised December 5, 2017; accepted January 2, 2018; published May 31, 2018

\begin{abstract}
Considering a dual-hop energy-harvesting (EH) relaying system, this paper advocates novel relaying protocols based on adaptive time power switching-based relaying (AR) architecture for amplify-and-forward (AF) mode. We introduce novel system model relaying network with impacts of co-channel interference (CCI) and derive analytical expressions for the average harvested energy, outage probability, and the optimal throughput of the information transmission link, taking into account the effect of CCI from neighbor cellular users. In particular, we consider such neighbor users procedure CCI both on the relay and destination nodes. Theoretical results show that, in comparison with the conventional solutions, the proposed model can achieve optimal throughput efficiency for sufficiently small threshold SNR with condition of reasonable controlling time switching fractions and power splitting fractions in concerned AR protocol. We also explore impacts of transmission distances in each hop, transmission rate, the other key parameters of AR to throughput performance for different channel models. Simulation results are presented to corroborate the proposed methodology.
\end{abstract}

Keywords: Co-channel interference, energy harvesting , throughput, adaptive relaying 


\section{Introduction}

Together with the rapid growth of services and applications in wireless communications, the demand for increasing power consumption in wireless terminals has dramatically enlarged. Motivated from advantages of various energy harvesting (EH) architectures, relaying networks have now been proposed to improve the overall system energy efficiency through considering information transmission and energy-transmission cooperation. The popular energy resources in EH including solar and wind harvest that is intermittent under impacts of the environmental alteration, radio frequency (RF) signal is considered as a new viable source which can be applied in wireless power transfer (WPT). Recently, the huge number of terminals with the enormous traffic load has increased the need for higher data rates and capacity. Recent years, one of the key technologies for future 5G wireless communications is that EH enabled relaying network. The main duty in such topology, to expand the coverage of wireless networks relay node can forward signal to the destination node from the source with helping of EH-asissted relay. As a result, combining EH and relaying scheme has received significant considerations from the research community and it is nominated as a favorable solution to resolve the energy shortage difficulties in energy-constrained wireless networks [1-4].

In [5], an ambitious switching policy was taken into account, in which the EH relay node switches between data relaying and $\mathrm{EH}$ function depending on whether or not the remaining energy at the relay node make sure that decoding at the destination is successful. The authors in [6] considered multiple source-destination pairs and a EH relay in terms of a cooperative network. Since a large-scale approach of radio-frequency (RF)-EH was implemented in wireless system, a network performance with random quantity of transmitter-receiver pairs was investigated straightforwardly with the help of stochastic geometry. Furthermore, a harvest-then-cooperate (HTC) protocol relying on the time-switching (TS) based EH architecture for an AF relaying network was considered by Chen et. al. [7], where during the energy downlink phase is scavenged by the source and the relay from the access point and they also assist the source's information transmission during the uplink phase. In [8], several metrics such as ergodic capacity and the outage probability of a multi-antenna relay system under the impact of co-channel interference (CCI) have been studied, where the exploitation of CCI is considered as a promising source of wireless energy. According to aforementioned studies, in EH relay systems, the operation mode of the wireless powered terminals [5] or the scheduling of relay nodes [7] is not similar to that of traditional cooperative systems. Mainly because scavenged energy at EH receiver relies solely on the instantaneous channel gains from the source's RF radiation, meaning that the system performance can be greatly enhanced by the dynamic exploitation of wireless fading variance in RF powered systems. By designating the cooperative role of each node dynamically within cooperative relaying systems, a substantially better diversity gain can be derived, which was referred to as role selection (ROSE) cooperation [8].

In another line of research, in [9]-[11], they assumed that deterministic EH model in which the design system has exact information of the energy arrival time and the amount of harvested energy, regarding model of EH relay systems, there were some power allocation policies given. Nevertheless, due to the random energy arrival time and the amount of harvested energy, the deterministic EH model seems to be evaluated in related systems. Furthermore, under general energy harvesting profiles, the authors in [12]-[15] proposed a number of transmission 
policies. Particularly, because the stationarity and erodicity of the energy harvesting process, the joint relay selection was introduced and power allocation schemes are investigated [12]. Likewise, in [13], [14], since during any time of data transmission, energy can be scavenged, several power allocation schemes for cooperative EH networks were put forward. The study in [15] based on what have been accomplished in [13] and [14] to expand the buffer-aided link adaptive EH relay system, in which the change of EH rates can be positively assisted. However, harvesting energy networks from resources in nature are the main focus in [15]. Recently, in terms of communication scenarios, WPT, which is a state-of-the-art technology has attracted extensive interests [16]-[19]. In such technology, there are several ways that energy can be scavenged 1) strongly coupled magnetic resonances, or 2) radio frequency (RF) signals. However, relying on magnetic resonances energy transfer is limited to some extent. For instance, energy receiver is required to install a coil tuned in order to resonate at precisely the similar frequency to the coil on the energy transmitter, and near field induction actuates energy transfer from strong nodes, namely base stations and vehicles. In comparison with strongly coupled magnetic resonances, energy transfer based on RF signals transcends the aforementioned limitations and energy and data can be transmitted by RF signals simultaneously apparently. Therefore, a system model is introduced, namely simultaneous wireless information and power transfer (SWIPT), which is also a RF-based energy harvesting method, has attracted increasing interest, as energy can be harvested from RF signals and the carried information is processed at the same time by network nodes [16] with the help of SWIPT. The investigation in [16] also presented the concept of SWIPT for point-to-point communication networks while the research results in [16] was continuously expanded in [17] to consider frequency-selective channels. Nonetheless, a robust design of receiver is the primary focus of the studies in [16] and [17], in which power from the same received signal can be derived and seen concurrently and more importantly the carried information cannot be decoded directly by practical circuits for harvesting energy [18]. Therefore, there was a proposed model for a robust receiver design in [18], [19] with separate information decoding and energy harvesting receivers, where receivers for information decoding and energy harvesting are operated in a time switching or power splitting manner.

However, in multi-cell cellular wireless system, co-channel interference and related research challenges are studied [20, 21]. Even though the proposed protocols in [22] the outage probability and the average throughput are investigated under the effect of CCI on the wireless powered communications, the energy efficiency still need be improved in EH protocol. Together with novel scheme presented in [23], an adaptive receiving architecture was proposed based on main idea of combining the time switching based relaying (TSR) and power splitting based relaying (PSR) receivers [24] in order to obtain optimal performance. Such adaptive energy harvesting relaying design is established to permit obtaining both energy and information at the relay. Motivated by the these discussions, in this paper we propose and analyse a relaying system with WPT under collecting CCI at the relay as an external energy resource. Our results show that the TS factor and the transmission power at the relay should be jointly designed to achieve an optimal throughput efficiency, and that optimizing the TS factor alone is inadequate as performed in the literature. The main contributions of this paper can be shown as:

i). The new architecture related to adaptive EH (namely AR protocol) is investigated and the impacts of CCI on system performance are studied. Such system model is designed as combination of the two traditional EH receivers TSR and PSR in one adaptive receiver. 
ii). We derive some analytical expressions of cumulative distributation function (CDF) of signal-to-interference-plus-noise ratio (SINR) and then throughput is derived for both delay-limited transmission (DLT) and delay-tolerant transmission (DTT) modes with considerations of CCI and AR protocol applied at the relay.

iii). Next, Monte-Carlo simulations are presented the throughout to corroborate our analysis and the impacts of some significant CCI parameters on AR protocol in EH assisted networks are investigated.

The remainder of this paper is organized as follows: Section 2 presents the system model and AR protocol is investigated in Section 3. In section 4, we derive the analytical expressions of outage probability and throughput in DLT abd DTT modes. Section 5 examines the simulation results. Finally, Section 6 completes with conclusion remarks for the paper and reviews the important results.

\section{System model}

We consider cooperative AF relaying network, where the source $(S)$ communicates with the destination $(D)$ through an intermediate relay $(R)$. The link between the source and the destination is unreliable or unavailable, so the transmission can only happen successfully with the aid of the relay. In this study, the deployed relay node is characterized as energy-constrained equipment. Furthermore, we assume a single antenna operated in the half-duplex mode is equipped in each node to prevent the simultaneous transmission and reception in the same frequency among nodes. The channel gains between the nodes are modeled as $h_{S R} \sim \mathcal{C N}\left(0, \Omega_{S R}\right)$ and $h_{R D} \sim \mathcal{C N}\left(0, \Omega_{R D}\right)$, assumed that all channels follow flat fading Rayleigh distribution.

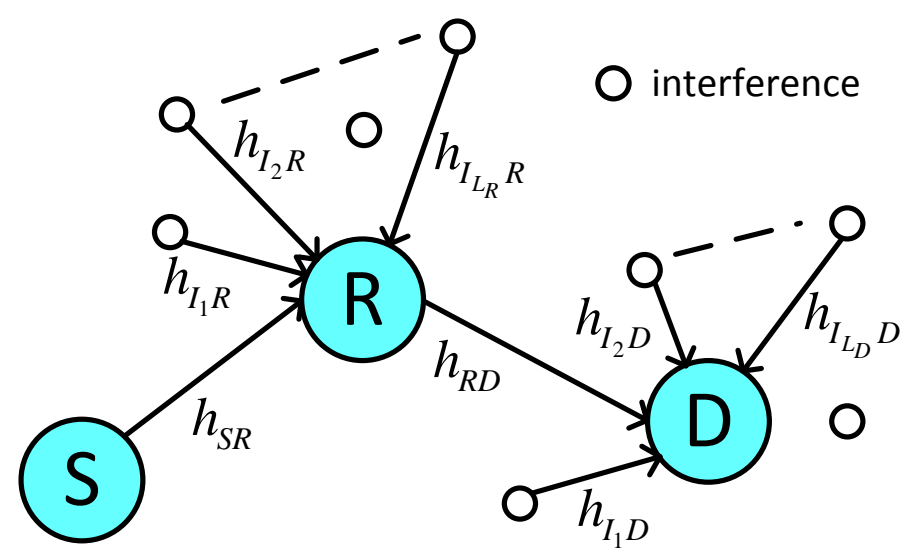

Fig. 1. System model with energy harvesting relay and co-channel interferences.

As illustrated in Fig. 1, the network under this investigation is affected by CCI signals, where $h_{I_{i} \mathrm{X}} \sim \mathcal{C N}\left(0, \Omega_{I_{i} \mathrm{X}}\right)$ is the complex channel fading gain between the $i^{t h}$ interferer and node $\mathbf{X}$, where $\mathbf{X} \in\{R, D\}$. In the presence of CCI signals, the overall performance of the system can be deteriorated. However, with the help of harvesting mechanism at the relay, CCI 
signals become extra sources of energy and can be consumed to support the relay-to-destination transmission. We assume that all channels, the desired channels and interference channels, are independent from each other. Moreover, the distance between node $i$ and node $j$ is denoted by $d_{i j}$, and $m>2$ is the path loss exponent.

\section{Adaptive Relaying (AR) Protocol}

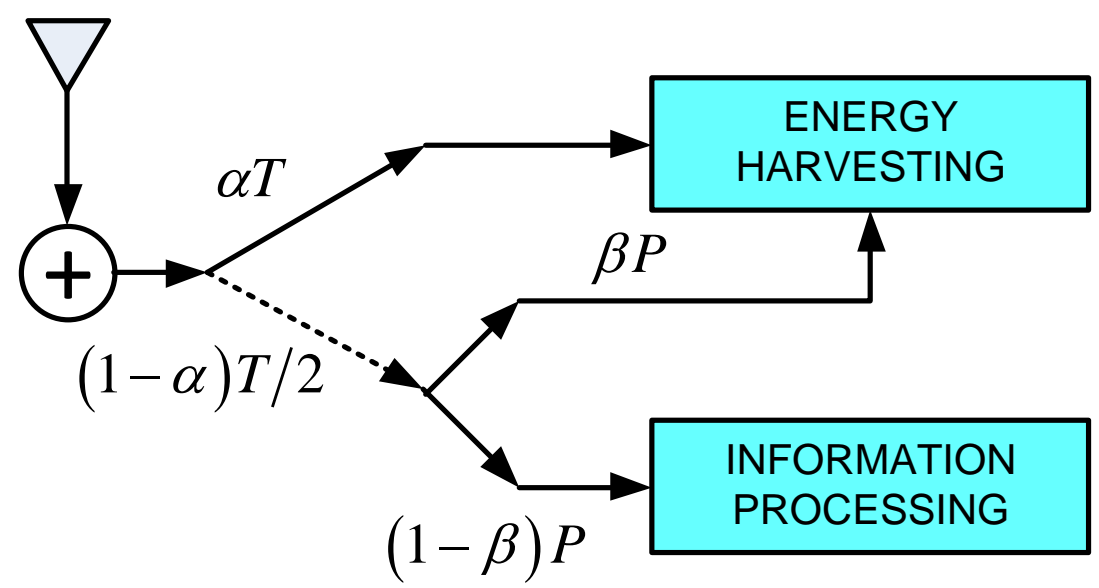

Fig. 2. Adaptive relaying architecture for energy harvesting.

In this paper, the relay harvests energy from the information signal and the CCI signals based on AR protocol [23]. The relay utilizes the harvested energy to amplify the received signal and then forward the amplified signal to the destination. The block time model illustrated in Fig. 2 presents the key parameters of AR protocol, where $T$ is the block time in which a certain block of information is transmitted from source to destination. The harvesting process occurs during $\alpha T$ block time, in which $0<\alpha<1$. The remaining block time is equally divided into two parts, that is $(1-\alpha) T / 2$. The first part is used for the source-to-relay transmission and the second part is allocated for the relay-to-destination transmission. Unlike TSR protocol [4], in the AR protocol, the relay also harvests a fraction of the received power, i.e. $\beta P$ $(0<\beta<1)$, the remaining power, $(1-\beta) P$, is utilized for information processing. Notice that, as $\beta \rightarrow 0$ (or $\alpha \rightarrow 0$ ) the above model shares the same function as TSR model (or PSR model).

Aforementioned, due to the presence of CCI signals, the received signal at the relay node, $y_{R}$ is given by:

$$
y_{R}=\sqrt{(1-\beta) \frac{P_{S}}{d_{S R}^{m}}} h_{S R} S_{S}+\sqrt{1-\beta} \sum_{i=1}^{L_{R}} \sqrt{\frac{P_{I_{i} R}}{d_{I_{i} R}^{m}}} h_{I_{i} R} S_{I_{i} R}+n_{R}
$$


where $S_{S}$ is the information signal with power of $P_{S}=\mathbb{E}\left\{\left|S_{S}\right|^{2}\right\}, d_{I_{i} R}$ is the distance between the $i^{\text {th }}$ interferer and the relay, $S_{I_{i} R}$ denotes the $i^{\text {th }}$ CCI signal with power of $P_{I_{i} R}=\mathbb{E}\left\{\left|S_{I_{i} R}\right|^{2}\right\}, L_{R}$ is the number CCI signals affecting the relay, $n_{R}$ is the additive white Gaussian noise (AWGN) with zero mean and variance of $\sigma_{R}^{2}$ at the relay. In this paper, the worst case is examined, where the power splitting scales down the signal power but the noise power remains unchanged. This scenario provides a lower-bound measurement for relaying networks in practice. Accordingly, after activated, an amount of block time, $\alpha T$ is spent by the relay for harvesting energy, and thus, the harvested energy is obtained at:

$$
E_{H}=\eta\left(\alpha+\frac{(1-\alpha) \beta}{2}\right)\left(\frac{P_{S}}{d_{S R}^{m}}\left|h_{S R}\right|^{2}+\sum_{i=1}^{L_{R}} \frac{P_{I_{i} R}}{d_{I_{i} R}^{m}}\left|h_{I_{i} R}\right|^{2}\right) T
$$

where $\eta(0 \leq \eta \leq 1)$ is the energy conversion efficiency. Since the energy harvested from AWGN noise is significantly small, it can be neglected for simplicity. The relay then consume the harvested energy and utilize that energy to support relay to destination information transmission. Therefore, the transmit power at the relay is given by:

$$
P_{R}=\frac{E_{H}}{(1-\alpha) T / 2}=\theta\left(\frac{P_{S}}{d_{S R}^{m}}\left|h_{S R}\right|^{2}+\sum_{i=1}^{L_{R}} \frac{P_{I_{i} R}}{d_{I_{i} R}^{m}}\left|h_{I_{i} R}\right|^{2}\right) \eta
$$

where $\theta=(2 \alpha /(1-\alpha))+\beta$. Consequently, the relay amplifies and forwards the amplified signal to the destination. It's worth noting that CCIs contributes as external interference to destiantion node. Hence, the received signal at the destination is given by:

$$
y_{D}=\frac{y_{R}}{\sqrt{d_{R D}^{m}}} h_{R D} G+\sum_{j=1}^{L_{D}} \sqrt{\frac{P_{I_{j} D}}{d_{I_{j} D}^{m} D}} h_{I_{j} D} S_{I_{j} D}+n_{D}
$$

where $n_{D}$ is the AWGN with zero mean and variance of $\sigma_{D}^{2}, d_{I_{j} D}$ is the distance between the $j^{\text {th }}$ interferer and the destination, $S_{I_{j} D}$ denotes the $j^{\text {th }}$ CCI signal with power of $P_{I_{j} D}=\mathbb{E}\left\{\left|S_{I_{j} D}\right|^{2}\right\}, L_{D}$ is the number CCI signals affecting the destination, $G$ is the gain factor and can be expressed as:

$$
G=\sqrt{P_{R}}\left((1-\beta) \frac{P_{S}}{d_{S R}^{m}}\left|h_{S R}\right|^{2}+(1-\beta) \sum_{i=1}^{L_{R}} \frac{P_{I_{i} R}}{d_{I_{i} R}^{m}}\left|h_{I_{i} R}\right|^{2}+\sigma_{R}^{2}\right)^{-1 / 2}
$$


For brevity in throughput analysis, the noise power term at the relay, $\sigma_{R}^{2}$, is assumed to be significantly small compared with the main signal power. Under this high transmit power approximation, the gain factor becomes:

$$
\begin{aligned}
G & \simeq \sqrt{P_{R}\left((1-\beta) \frac{P_{S}}{d_{S R}^{m}}\left|h_{S R}\right|^{2}+(1-\beta) \sum_{i=1}^{L_{R}} \frac{P_{I_{i} R}}{d_{I_{i} R}^{m}}\left|h_{I_{i} R}\right|^{2}+\sigma_{R}^{2}\right)^{-1 / 2}} \\
& =\sqrt{\frac{\theta\left(\frac{P_{S}}{d_{S R}^{m}}\left|h_{S R}\right|^{2}+\sum_{i=1}^{L_{R}} \frac{P_{I_{i} R}}{d_{I_{i} R}^{m}}\left|h_{I_{i} R}\right|^{2}\right) \eta}{(1-\beta) \frac{P_{S}}{d_{S R}^{m}}\left|h_{S R}\right|^{2}+(1-\beta) \sum_{i=1}^{L_{R}} \frac{P_{I_{i} R}}{d_{I_{i} R}^{m}}\left|h_{I_{i} R}\right|^{2}+\frac{\sigma_{R}^{2}}{P_{R}}}} \\
& =\sqrt{\frac{\theta\left(\frac{P_{S}}{d_{S R}^{m}}\left|h_{S R}\right|^{2}+\sum_{i=1}^{L_{R}} \frac{P_{I_{i} R}}{d_{I_{i} R}^{m}}\left|h_{I_{i} R}\right|^{2}\right) \eta}{(1-\beta) \frac{P_{S}}{d_{S R}^{m}}\left|h_{S R}\right|^{2}+\left.(1-\beta) \sum_{i=1}^{L_{R}} \frac{P_{I_{i} R}}{d_{I_{i} R}^{m}} h_{I_{i} R}\right|^{2}+\frac{\sigma_{R}^{2}}{P_{R}}}} \\
& \simeq \sqrt{\frac{\eta \theta}{1-\beta}}
\end{aligned}
$$

Subsequently, by substituting (1) into (4), the received signal at the destination can also be expressed as:

$$
\begin{aligned}
y_{D} & =\sqrt{(1-\beta) \frac{P_{S}}{d_{S R}^{m}}} h_{S R} \frac{h_{R D}}{\sqrt{d_{R D}^{m}}} G s_{S} \\
& +\left(\sqrt{1-\beta} \sum_{i=1}^{L_{R}} \sqrt{\frac{P_{I_{i} R}}{d_{I_{i} R}^{m}}} h_{I_{i} R} S_{I_{i} R}+n_{R}\right) \frac{h_{R D}}{\sqrt{d_{R D}^{m}}} G+\sum_{j=1}^{L_{D}} \sqrt{\frac{P_{I_{j} D}}{d_{I_{j} D}^{m}}} h_{I_{j} D} S_{I_{j} D}+n_{D}
\end{aligned}
$$

By assuming that $\sigma_{R}^{2}=\sigma_{D}^{2}=\sigma^{2}$, the received signal-to-interference-plus-noise ratio (SINR) at the relay under high SNR approximation is obtained as:

$$
\begin{aligned}
\gamma_{D} & =\frac{(1-\beta) \frac{P_{S}}{d_{S R}^{m}}\left|h_{S R}\right|^{2} \frac{\left|h_{R D}\right|^{2}}{d_{R D}^{m}} G^{2}}{\left.\left((1-\beta) \sum_{i=1}^{L_{R}} \frac{P_{I_{i} R}}{d_{I_{i} R}^{m}}\left|h_{I_{i} R}\right|^{2}+\sigma^{2}\right)\left|\frac{\left.h_{R D}\right|^{2}}{d_{R D}^{m}} G^{2}+\sum_{j=1}^{L_{D}} \frac{P_{I_{j} D}}{d_{I_{j} D}^{m}}\right| h_{I_{j} D}\right|^{2}+\sigma^{2}} \\
& =\frac{\gamma_{S R} \gamma_{R D}}{\left(I_{R}+1\right) \gamma_{R D}+\left(I_{D}+1\right)}
\end{aligned}
$$


where $\gamma_{S R}=(1-\beta)\left|h_{S R}\right|^{2} P_{S} / d_{S R}^{m} \sigma^{2}, I_{R}=(1-\beta) \sum_{i=1}^{L_{R}}\left|h_{I_{i} R}\right|^{2} P_{I_{i} R} / \sigma^{2} d_{I_{i} R}^{m}, \quad \gamma_{R D}=G^{2}\left|h_{R D}\right|^{2} / d_{R D}^{m}$ and $I_{D}=\sum_{j=1}^{L_{D}}\left|h_{I_{j} D}\right|^{2} P_{I_{j} D} / \sigma^{2} d_{I_{j} D}^{m}$.

\section{Throughput Analysis}

\subsection{Outage Capacity and the Achievable Throughput}

Considering on DLT where the destination can interpret the received signal by transmission as block by block. As a result, in DLT code length could not be longer than the the block time for signal transmission. In this case, the source conveys data under the fixed rate and we first examine the outage probability to obtain the average throughput as below expression

$$
C_{O}=\left(1-P_{\text {out }}\right) R
$$

where $P_{\text {out }}=F_{\gamma_{D}}\left(\gamma_{0}\right), \gamma_{0}=2^{R}-1$ and $R$ stands for the transmisson rate of the soure node. The throughput in the delay limited mode is achieved by:

$$
\tau_{O}=\frac{(1-\alpha) C_{O}}{2}
$$

It is noted that the optimal throughput is achieve by solving $\tau_{O}^{\text {optimal }}=\underset{(\alpha ; \beta)}{\operatorname{argmax}}(1-\alpha) C_{O} / 2$. However, since the derivation of the solution of the optimal throughput requires many complex computational steps, therefore for brevity, $\tau_{O}^{\text {optimal }}$ is obtained by simply applying exhaustive search method.

\subsection{CDF Calculation of SINR}

In this section, the CDF of SINR can be calculate to find the throughput corresponding DLT and DTT approaches as follow:

Theorem 1: Under high SNR approximation condition, the CDF of the received SINR at the destination, $F_{\gamma_{D}}^{A P}$ is given by: 


$$
\begin{aligned}
& F_{\gamma_{D}}^{A P}(\gamma)=1-\exp \left(-\frac{\gamma}{\lambda_{S R}}\right)\left\{\sum_{i=1}^{n^{R}} \sum_{j=1}^{m_{i}^{R}} \Phi_{i j}^{R}\left(\frac{v_{[i]}}{\lambda_{S R}} \gamma+1\right)^{-j}\right\} \\
& \times\left\{\sum_{i=1}^{n^{D}} \sum_{j=1}^{m_{i}^{D}} \sum_{n=0}^{j-1}\left(\begin{array}{c}
j-1 \\
n
\end{array}\right) \Phi_{i j}^{D} \frac{v_{[i]}^{-j} \mathrm{e}^{1 / \iota_{i]}}}{(j-1) !}(-1)^{j-n-1}\right. \\
& \left.\times 4 \sqrt{\frac{\gamma}{\lambda_{S R} \lambda_{R D}}} \int_{1}^{\infty} \kappa^{2 n+2} \exp \left(-\frac{\kappa^{2}}{v_{[i]}}\right) K_{1}\left(2 \sqrt{\frac{\gamma}{\lambda_{S R} \lambda_{R D}}} \kappa\right) d \kappa\right\}
\end{aligned}
$$

where $\lambda_{S R}=(1-\beta) P_{S} \Omega_{S R} / d_{S R}^{m} \sigma^{2}$ and $v_{i}=(1-\beta) P_{I_{i} X} \Omega_{I_{i} \mathrm{X}} / d_{I_{i} \mathrm{X}}^{m} \sigma^{2}$. This theorem is achieved with the help of the probability density function (PDF) of $I_{\mathrm{X}}$, which is given by:

$$
f_{I_{\mathrm{X}}}(\gamma)=\sum_{i=1}^{n^{\mathrm{X}}} \sum_{j=1}^{m_{i}^{\mathrm{X}}} \Phi_{i j}^{\mathrm{X}} \frac{v_{[i]}^{-j}}{(j-1) !} \gamma^{j-1} \exp \left(-\frac{\gamma}{v_{[i]}}\right)
$$

where $\Phi_{i j}^{\mathbf{X}}$ is the $(i, j)$ th characteristic coefficient of the diagonal matrix $\mathcal{X}_{\mathrm{X}}=\operatorname{diag}\left(v_{1}, v_{2}, \ldots, v_{L_{\mathrm{X}}}\right), n^{\mathrm{x}}$ is the number of distinct diagonal elements of $\mathcal{X}_{\mathrm{x}}$, $v_{[i]}>v_{[i+1]}>\ldots>v_{\left[n^{\mathrm{x}}\right]}$ are the distinct elements in decreasing order and $m_{i}^{\mathrm{X}}$ is the multiplicity of $v_{i}$ [22]. When the interfering signals are statistically independent and identically distributed (i.i.d.), i.e., $v_{i}=v_{\mathrm{X}}$, then $n^{\mathrm{X}}=1, m_{i}^{\mathrm{X}}=L_{\mathrm{X}}$; the PDF of $I_{\mathrm{X}}$ becomes:

$$
f_{I_{\mathbf{X}}}(\gamma)=\frac{v_{\mathbf{X}}^{-L_{\mathbf{X}}}}{\left(L_{\mathbf{X}}-1\right) !} \gamma^{L_{\mathbf{X}}-1} \exp \left(-\frac{\gamma}{v_{\mathbf{X}}}\right)
$$

In addition, when all of $v_{1}$ 's are distinct, i.e. $n^{\mathrm{X}}=L_{\mathrm{X}}, m_{i}^{\mathrm{X}}=1$; the PDF of $I_{\mathrm{X}}$ becomes:

$$
f_{I_{\mathrm{X}}}(\gamma)=\sum_{i=1}^{L_{\mathrm{X}}}\left(\prod_{\substack{k=1 \\ k \neq i}}^{L_{\mathrm{X}}} \frac{1}{v_{i}-v_{k}}\right) \exp \left(-\frac{\gamma}{v_{i}}\right)
$$

Proof: See appendix A.

Proposition 1: We consider two scenario, the case 1 as only one CCI signal affects R and D or the case 2 corresponding the interferences are i.i.d., (12) can be simplified as follow: 


$$
\begin{aligned}
F_{\gamma_{D}}^{A P}(\gamma)= & -\exp \left(-\frac{\gamma}{\lambda_{S R}}\right)\left(\frac{v_{R}}{\lambda_{S R}} \gamma+1\right)^{-L_{R}} \\
& \times\left\{4 \frac{v_{D}^{-L_{D}} \mathrm{e}^{1 / v_{D}}}{\left(L_{D}-1\right) !} \sqrt{\frac{\gamma}{\lambda_{S R} \lambda_{R D}}} \sum_{n=0}^{L_{D}-1}\left(\begin{array}{c}
L_{D}-1 \\
n
\end{array}\right)(-1)^{L_{D}-n-1}\right. \\
& \left.\times \int_{1}^{\infty} \kappa^{2 n+2} \exp \left(-\frac{\kappa^{2}}{v_{D}}\right) K_{1}\left(2 \sqrt{\frac{\gamma}{\lambda_{S R} \lambda_{R D}}} \kappa\right) d \kappa\right\}
\end{aligned}
$$

Denote $\overline{\mathrm{SNR}} \triangleq P_{S} \Omega_{S R} / \sigma^{2}$ as the average signal-to-noise ration (SNR) and $\overline{\mathrm{SIR}}_{\mathrm{X}} \triangleq P_{S} \Omega_{S R} / \sum_{i=1}^{L_{\mathrm{X}}} P_{I_{i} \mathrm{X}} \Omega_{I_{i} \mathrm{X}}$ as the average signal-to-interference ration (SIR). It is worth noting that in order to establish the i.i.d. case when $L_{\mathbf{X}} \geq 2, d_{I_{i} \mathbf{X}}$ and $P_{I_{i} \mathbf{X}}$ must sastify the following constrains:
a) $\frac{P_{I_{1} X} \Omega_{I_{1} X}}{d_{I_{1} X}^{m}}=\frac{P_{I_{2} X} \Omega_{I_{2} X}}{d_{I_{2} X}^{m}}=\ldots=\frac{P_{I_{L_{X}} \mathrm{X}} \Omega_{I_{L_{X} X} \mathrm{X}}}{d_{I_{L_{X}} \mathrm{X}}^{m}}$ and
b) $\sum_{i=1}^{L_{\mathrm{X}}} \frac{P_{I_{i} \mathrm{X}} \Omega_{I_{i} \mathrm{X}}}{\sigma^{2}}=\frac{\overline{\mathrm{SNR}}}{\overline{\mathrm{SIR}_{\mathrm{X}}}}$

Given the values of all $d_{I_{\mathrm{i}} \mathrm{X}}, P_{I_{\mathrm{i}} \mathrm{X}}$ can be simply obtained as

$$
P_{I_{i} X} \Omega_{I_{i} X}=\frac{d_{I_{i} \mathrm{X}}^{m}}{\sum_{i=1}^{L_{\mathrm{X}}} d_{I_{i} \mathrm{X}}^{m}} \frac{\overline{\mathrm{SNR}}}{\overline{\operatorname{SIR}_{\mathrm{X}}}}
$$

It is noted that channel condition i.i.d. when $\Omega_{I_{1} \mathrm{X}}=\Omega_{I_{2} \mathrm{X}}=\ldots=\Omega_{I_{L_{X}} \mathrm{X}}$, occurs in a scenario where the near interferer makes smaller power transmitted to the affected node compared with the far interferer. It means that the closer the interference is, the smaller power its interfered node receive. A more realistic scenario is given by Proposition 2 .

Proposition 2: when $L_{\mathrm{x}} \geq 2$ and the interferences are independent and non-identically distributed (i.n.d.), we then have a new expression for (12) as: 


$$
\begin{aligned}
F_{\gamma_{D}}^{A P}(\gamma)= & -\exp \left(-\frac{\gamma}{\lambda_{S R}}\right)\left\{\sum_{i=1}^{L_{R}}\left[\prod_{\substack{k=1 \\
k \neq i}}^{L_{R}} \frac{v_{i}}{v_{i}-v_{k}}\right]\left(\frac{v_{i}}{\lambda_{S R}} \gamma+1\right)^{-1}\right\} \\
& \times\left\{\sum_{i=1}^{L_{D}}\left[\prod_{\substack{k=1 \\
k \neq i}}^{L_{D}} \frac{v_{i}}{v_{i}-v_{k}}\right] \frac{4 \mathrm{e}^{1 / v_{i}}}{v_{i}} \sqrt{\frac{\gamma}{\lambda_{S R} \lambda_{R D}}}\right. \\
& \left.\times \int_{1}^{\infty} \kappa^{2} \exp \left(-\frac{\kappa^{2}}{v_{i}}\right) K_{1}\left(2 \sqrt{\frac{\gamma}{\lambda_{S R} \lambda_{R D}}} \kappa\right) d \kappa\right\}
\end{aligned}
$$

where $K_{1}($.$) is the first order modified Bessel function of the second kind [26].$

Proof: when the interferences are i.n.d. and all of the characteristic coefficient becomes [25]:

$$
\Phi_{i 1}^{\mathrm{X}}=\prod_{\substack{k=1 \\ k \neq i}}^{L_{\mathrm{X}}} \frac{v_{i}}{v_{i}-v_{k}}, \quad i=1,2, \ldots, L_{\mathrm{X}}
$$

Applying the condition for the i.n.d. case above, it can be obtained the CDF of SINR at the destination for the i.n.d. case. When $L_{\mathbf{X}}=0$, i.e. the system is unaffected by CCI signals, $F_{\gamma_{D}}^{A P}(\gamma)$ then becomes:

$$
F_{\gamma_{D}}^{A P}(\gamma)=1-2 \sqrt{\frac{\gamma}{\lambda_{S R} \lambda_{R D}}} K_{1}\left(2 \sqrt{\frac{\gamma}{\lambda_{S R} \lambda_{R D}}}\right) \exp \left(-\frac{\gamma}{\lambda_{S R}}\right)
$$

\subsection{Upper bounds on the CDF}

In this section, we define the upper-bound of the SINR at the destination node is defined as

$$
\gamma_{D}^{A P} \leq \gamma_{D}^{U P}=\frac{\gamma_{S R} \gamma_{R D}}{\left(I_{R}+1\right) \gamma_{R D}+I_{D}}
$$

Consequently, the upper-bound for $F_{\gamma_{D}}^{A P}(\gamma)$ in normal case, i.i.d. case and i.n.d. case is given by (22) and (23) respectively.

$$
\begin{aligned}
F_{\gamma_{D}}^{U P}(\gamma)=1 & -\Gamma\left(1+L_{D}\right)\left(\frac{v_{R}}{\lambda_{S R}} \gamma+1\right)^{-L_{R}} \exp \left(-\frac{\gamma}{\lambda_{S R}}\right) \\
& \times W_{-L_{D}, \frac{1}{2}}\left(\frac{v_{D}}{\lambda_{S R} \lambda_{R D}} \gamma\right) \exp \left(\frac{v_{D}}{\lambda_{S R} \lambda_{R D}} \frac{\gamma}{2}\right)
\end{aligned}
$$




$$
\begin{aligned}
F_{\gamma_{D}}^{U P}(\gamma)=1 & -\left\{\sum_{i=1}^{L_{R}}\left[\prod_{\substack{k=1 \\
k \neq i}}^{L_{R}} \frac{v_{i}}{v_{i}-v_{k}}\right]\left(\frac{v_{i}}{\lambda_{S R}} \gamma+1\right)^{-1} \exp \left(-\frac{\gamma}{\lambda_{S R}}\right)\right\} \\
& \times\left\{\sum_{i=1}^{L_{D}}\left[\prod_{\substack{k=1 \\
k \neq i}}^{L_{D}} \frac{v_{i}}{v_{i}-v_{k}}\right] W_{-1, \frac{1}{2}}\left(\frac{v_{i}}{\lambda_{S R} \lambda_{R D}} \gamma\right) \exp \left(\frac{v_{i}}{\lambda_{S R} \lambda_{R D}} \frac{\gamma}{2}\right)\right\}
\end{aligned}
$$

Proof: by letting the lower limit of the integral in the equation (16) and (18) to 1 and with help of (Eq. 6.643.3) in [27] the above results can be obtained.

\section{Numerical Results}

In this section, we assume $\sigma^{2}=0.01 \mathrm{~W}$ and $L_{R}=L_{D}=5$. In order to make the simulation process simpler, the average SNR is set to $30 \mathrm{~dB}$ for most figures; we also assume that $\overline{\mathrm{SIR}}_{\mathbf{X}}=$ $20 \mathrm{~dB}$ for most figures. The energy harvesting efficiency, $\eta$ is set to 1 ; the transmission rate at the source is set to 3 (bits/sec/Hz) and the path loss exponent is 2.4. In addition, the distances are normalized as $d_{S R}=1.5$ and $d_{R D}=2.0$ unless stated otherwise.

To evaluate the impacts of co-channel interference's power to the throughput of the sustem, we define $\left\|v_{\mathbf{X}}\right\| \triangleq\left[\rho_{1}, \rho_{2}, \ldots, \rho_{L_{\mathbf{X}}}\right]$, where $P_{I_{i} \mathbf{X}} \Omega_{I_{i} \mathbf{X}}=\rho_{i}(\overline{\operatorname{SNR}} / \overline{\operatorname{SIR}} \mathbf{X})$ and $\rho_{1}>\rho_{2}>\ldots$ $\ldots>\rho_{L_{\mathrm{X}}}$. Note that $\rho_{i}=1 / L_{\mathrm{X}}$ for i.i.d. case. Finally, for simplicity, we let $\Omega_{I_{i} \mathrm{X}}=\Omega_{S R}=1$ and $d_{I_{1} \mathrm{X}}=d_{I_{2} \mathrm{X}}=\ldots=d_{I_{L_{\mathrm{X}}} \mathrm{X}}$.
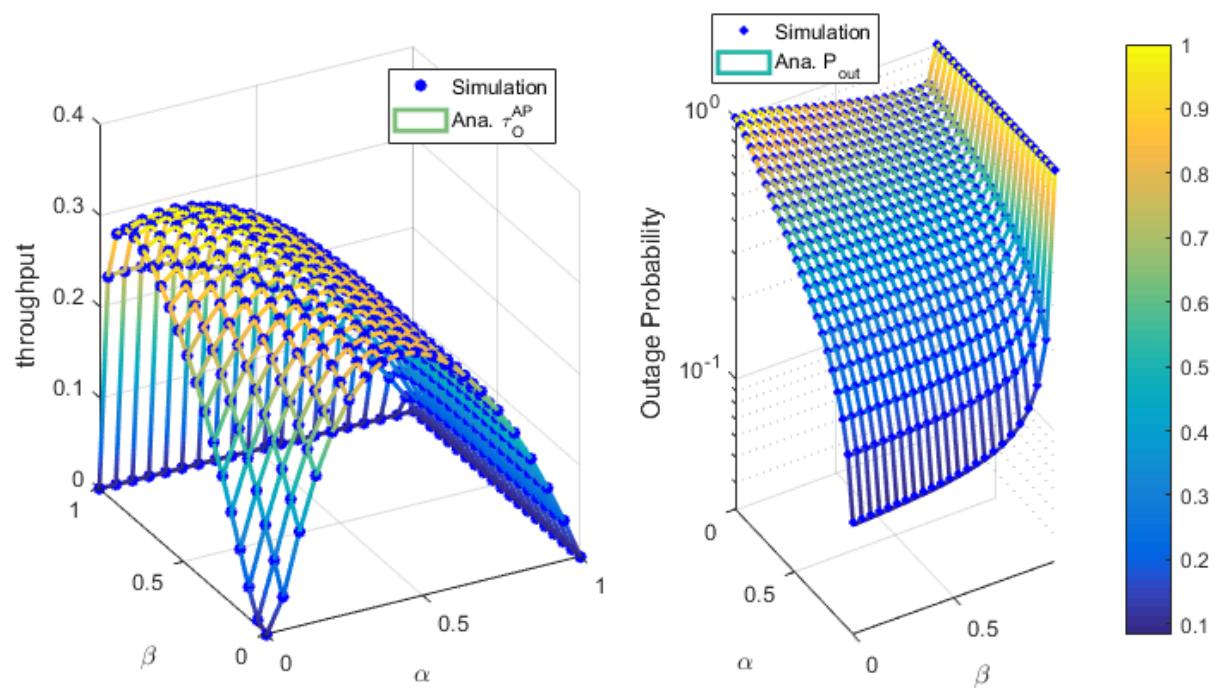

Fig. 3. Effect of time and power splitting coefficients on the throughput and outage performance. 
Fig. 3 plots the throughput versus EH time and power splitting parameters in AR protocol in the left side of the figure, while outage probability in AR protocol is illustrated in the right side. It can be show that Monte-Carlo simulation line trictly meets the anlytical line. For the left side of Fig. 3, it can be shown that throughput increases as $\alpha$ and $\beta$ increase from 0 to the optimal values, but later it starts decreasing as $\alpha$ and $\beta$ come cross its optimal values. The system performance changed remarkably when varying time switching from about 0.3 to 1 while power splitting fractions has small impact to throughput as $\beta \in(0.2,0.8)$. For the right side of this figure, the outage probability decreases as $\alpha$ increases, for each value of $\alpha$ it is observed that the outage probability decrease to a minimum value as $\beta$ and then if $\beta$ continues increases, the outage probability also goes up. Hence, it is shown that controlling time switching and power splitting fractions provides optimal throughput performance. It worth noting that deploying adaptive protocol in energy harvesting architecture is effecive way to find optimal system performance.

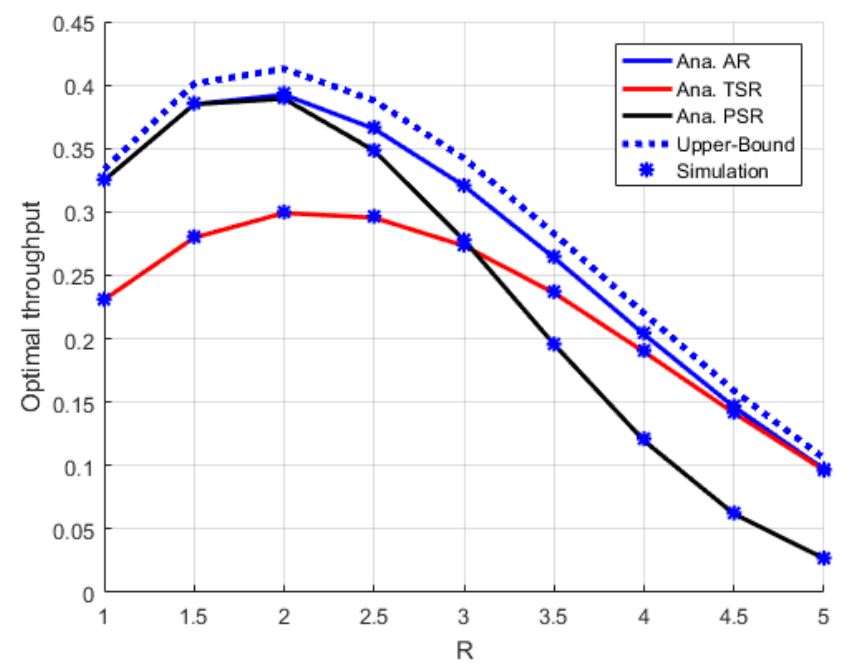

Fig. 4. Optimal throughput as varying of the source transmission rate, $R$.

As observation in Fig. 4, to study how the source transmission rate R can contribute to enhance on the optimal throughput performance by changing R from 2 to 6 as shown in Fig. 4 . It can be seen clearly that $\mathrm{R}$ improves the throughput firstly as the concerned rate go up to appropriate 3.5 with expected throughput and then higher rate leads to increasing the threshold SNR in calculation of outage probabilty. As a result, outage performce at higher rate will be worse and making the system throughput decreases significantly, especially in case of $R=5$ (bit/sec/Hz). It is noted that the AR in this scenario operates exactly as the case of PSR, after that the throughput starts decreasing as $\mathrm{R}$ increases further. In addition, the AR performs exactly as TSR case at high transmission rate. However, with medium value of the transition state (i.e. transmission rate changes from 3 to 5 (bit/sec/Hz), the AR-based system obtains higher throughput in comparison with both PSR and TSR. In addition, it can be seen that i.i.d channel contribute to better thoughput performance than i.i.d channel in corresponding protocol. 
Fig. 5a. and Fig. 5b. illutrate impact of distance on system performance. In particular, to explore the influence of $d_{S R}$ and $d_{R D}$ on the system throughput, one of them should be fixed as illustrated, and remaining distance changes in range from 1 to 5. Fig. 5a. and Fig. 5b. display the optimal throughput versus $\mathrm{d}_{\mathrm{SR}}$ and $\mathrm{d}_{\mathrm{RD}}$, respectively. As observation from the figure, the AR with optimal time/power allocation in energy harvesting architecture leads to gain better performance than PSR and TSR along with the distance increases from normalized one. The reasonable scenario is that adjusting the distance to small corresponding two nodes are closed positions and hence the relay needs little energy. This circumstance is equivalent with the AR employs exactly as PSR scheme, and there is no information missing. In constrast, in case of the distance rises, the PSR converts unable to harvest the required power. It can be concluded the AR allocates best time and power fraction to harvest energy, and hence the AR has larger throughput in comparison with the worsest case of PSR. The such performance gap can be seen clearly at maximal distance. It can be show that the optimal relay location is closer to the source to gain better performance.

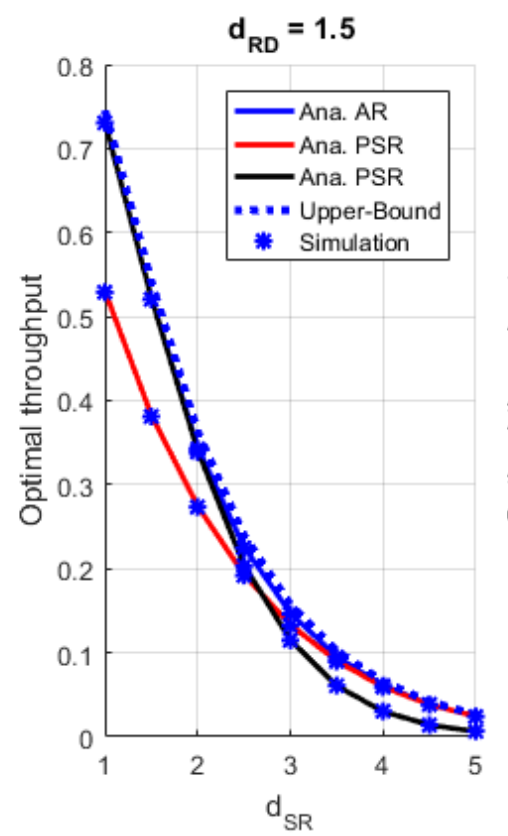

a) Effect of the first hop's distance.

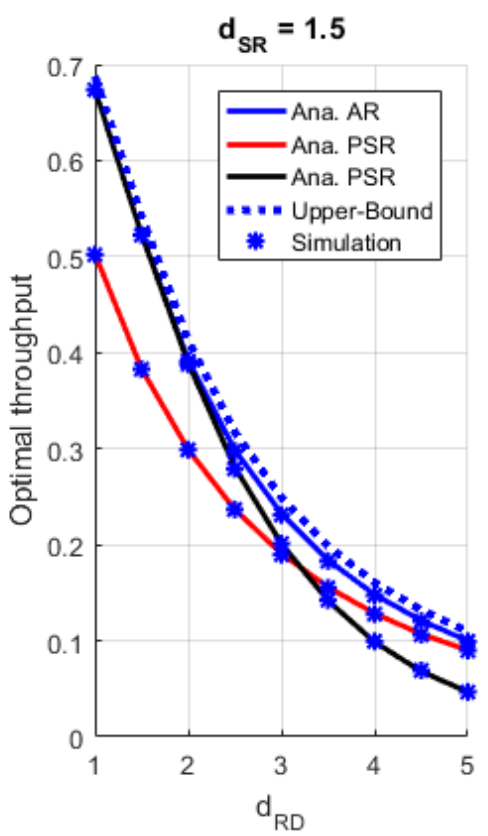

b) Effect of the second hop's distance.

Fig. 5. Impact of distance on the system throughput.

Next, Fig. 6. demonstrates the optimal throughput as varying the average SIR/SNR. As illustration in the figure, the optimal throughput increases as the average SIR/SNR increases. The main reason is that the higher power of the CCI components results in decreasing the average SIR/SNR and then it can weakens the system performance. The blue line clarifies the optimal throughput for AR protocol which is always outperforms TSR and PSR protocol. More importantly, the line graph showing AR and PSR protocol are greater performance compared with the TSR protocol, this trend is more clear at high SNR (i.e. SNR is greater than $15 \mathrm{~dB}$ ). It noted that we use specific value of the SNR in this simulation result for such evaluation. 

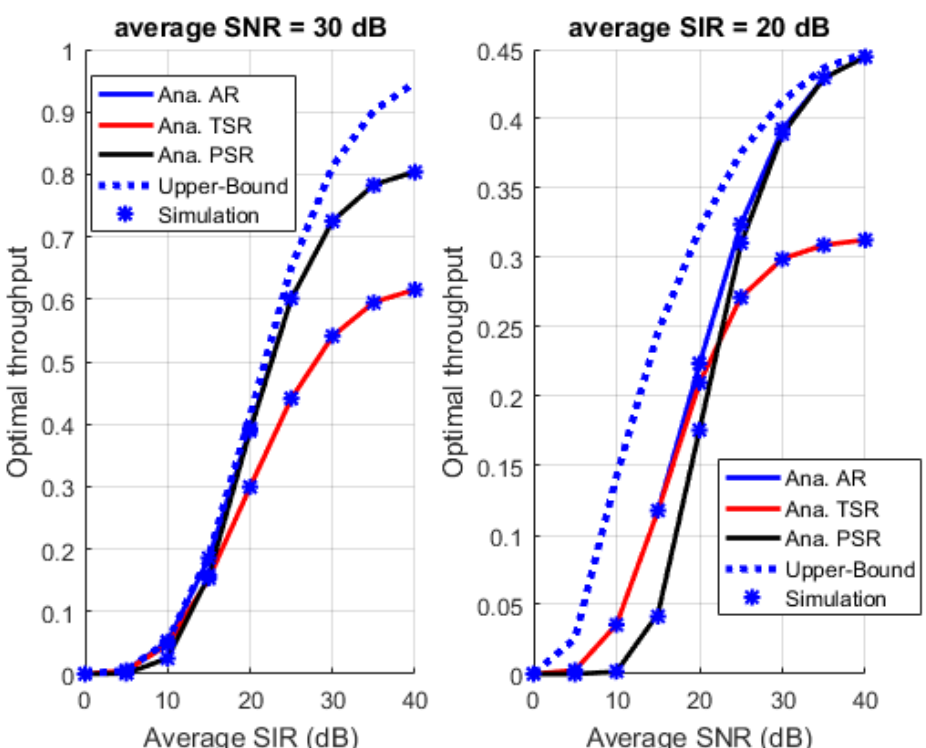

Fig. 6. Optimal throughput with varying the average SIR/SNR at source.

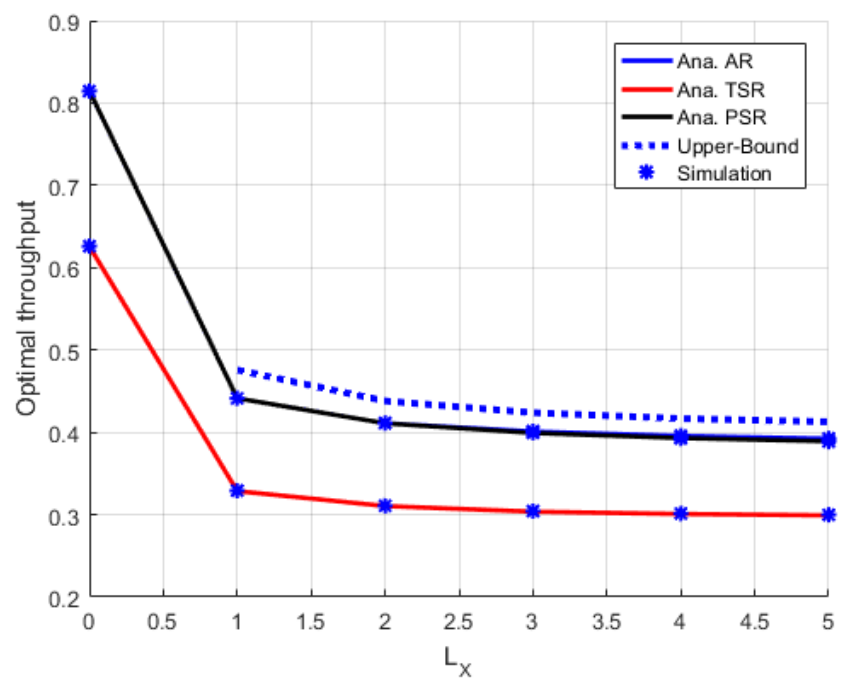

Fig. 7. Optimal throughput with the average SNR at source as varying the number of CCI sources.

Furthermore, in Fig. 7, it can be obtained the floor value of the throughput in range of the number of CCI sources from 2 to 6 . The similar trends of three protocols can be seen in this experiment, it confirms the advantage of AR for imroving throughput performance. In addition, the proposed system with non-CCI (in case of $L_{X}=0$ ) scheme gains better throughput as compared with CCI scheme. 


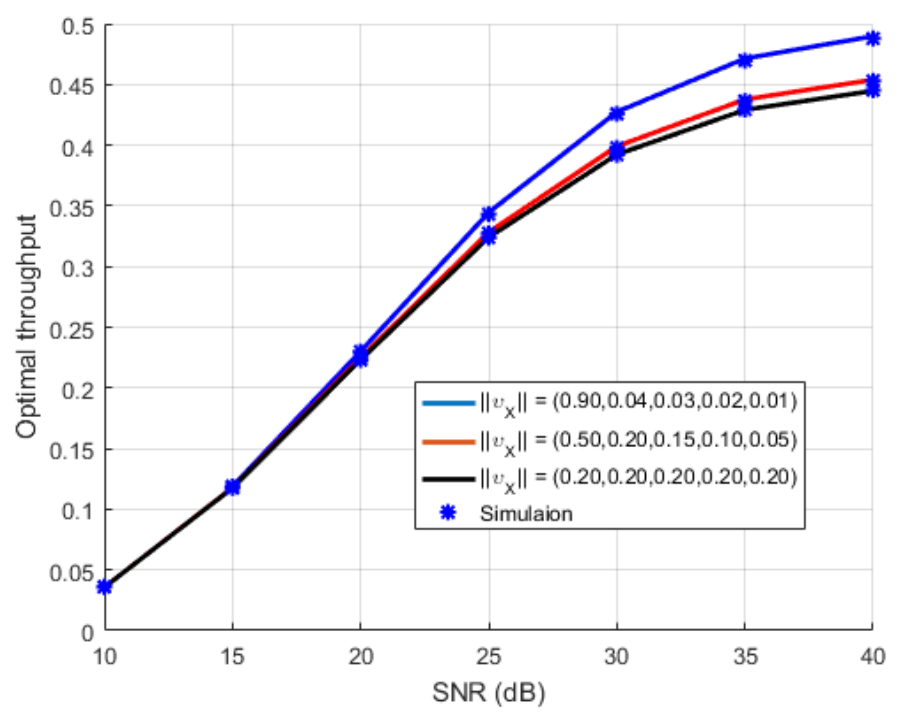

Fig. 8. Optimal throughput with varying the average SNR at source with different power distribution.

Considering on different $\left\|v_{\mathbf{x}}\right\|$ for the adaptive relaying protocol as in Fig. 8. The blue and red curves are obtained from i.n.d. case while the black one can be obtained from i.i.d. case. It is confirmed that different distribution of $P_{I_{i} X} \Omega_{I_{i} X}$ also plays an important role in the throughput of the system. Specifically, i.n.d. condition results in a better thoughput than that of i.i.d. case.

Remark 1: In general, one can observed from Fig. 4 to Fig. 7 that the upper-bound curves become approximal to the analytical curves under some specific circumstances. Consequently, we can effectively adopt (22) and (23) to measure system performance rather than (16) and (18), since the complexity of $F_{\gamma_{D}}^{A P}(\gamma)$ is higher than that of $F_{\gamma_{D}}^{U P}(\gamma)$ due to the integral parts.

\section{Conclusion}

This paper proposed and analyzed the performance of the energy harvesting-based relaying networks in the presence of CCI sources. The relay and destination node obtain more advantage from harvested energy from CCI as ambient RF sources. The explicit schemes are proposed at relaying network under impacts of CCI to increase the power transfer efficiency. By applying the propositions, the exact analytical expressions for the outage probability and the optimal throughput were derived. We showed that the system performance can be significantly improved by deploying AR protocol with optimal time and power allocation for energy harvesting receiver and multiple CCI sources for higher throughput in the proposed system model. 


\section{Appendix A}

PROOF OF THEOREM 1:

The CDF of random variable $\gamma_{D}$ can be expressed in probabilistic function as follow:

$$
\begin{aligned}
F_{\gamma_{D}}(\gamma)= & \mathbb{P}\left\{\frac{\gamma_{S R} \gamma_{R D}}{\left(I_{R}+1\right) \gamma_{R D}+\left(I_{D}+1\right)}<\gamma\right\} \\
= & 1-\mathbb{E}_{I_{R}, I_{D}, \gamma_{R D}}\left\{\exp \left(-\frac{\gamma}{\lambda_{S R}}\left[\left(I_{R}+1\right)+\left(\frac{I_{D}+1}{\gamma_{R D}}\right)\right]\right)\right\} \\
= & 1-\exp \left(-\frac{\gamma}{\lambda_{S R}}\right) \mathbb{E}_{I_{R}}\left\{\exp \left(-\frac{\gamma}{\lambda_{S R}} I_{R}\right)\right\} \\
& \times \mathbb{E}_{I_{D}, \gamma_{R D}}\left\{\exp \left(-\frac{\gamma}{\lambda_{S R}}\left(\frac{I_{D}+1}{\gamma_{R D}}\right)\right)\right\}
\end{aligned}
$$

The second equality is due to the CDF of $\gamma_{S R}$, i.e., $F_{\gamma_{S R}}(\gamma)=1-\exp \left(-\gamma / \lambda_{S R}\right)$ while (26) is achieved because $I_{R}, I_{D}$ and $\gamma_{R D}$ are independent random variables. The first expectation in (26) can be expressed as:

$$
\begin{aligned}
\mathbb{E}_{I_{R}}\left\{\exp \left(-\frac{\gamma}{\lambda_{S R}} I_{R}\right)\right\} & =\int_{0}^{\infty} \exp \left(-\frac{\gamma}{\lambda_{S R}} x\right) f_{I_{r}}(x) d x \\
& =\sum_{i=1}^{n^{R}} \sum_{j=1}^{m_{i}^{R}} \Phi_{i j}^{R} \frac{v_{[i]}^{-j}}{(j-1) !} \int_{0}^{\infty} x^{j-1} \exp \left[-\left(\frac{\gamma}{\lambda_{S R}}+\frac{1}{v_{[i]}}\right) x\right] d x \\
& =\sum_{i=1}^{n^{R}} \sum_{j=1}^{m_{i}^{R}} \Phi_{i j}^{R} \frac{v_{[i]}^{-j}}{(j-1) !}\left(\frac{v_{[i]}}{\lambda_{S R}} \gamma+1\right)^{-j}
\end{aligned}
$$

In the above expressions, (27) is obtained by using definition of expectation factor, i.e., $\mathbb{E}\{Y\}=\int_{0}^{\infty} y f_{Y}(y) d x$, where $\mathrm{Y}$ is a nonnegative random variable and $f_{Y}(y), y>0$ is its PDF. Furthermore, the last equality is derived with the help of identity (Eq. 2.3.3.1) in [27]. The second expectation in (26) can be calculated as follow

$$
\begin{aligned}
\mathbb{E}_{I_{D}, \gamma_{R D}}\{\ldots\} & =\int_{0}^{\infty} \int_{0}^{\infty} \exp \left[-\frac{\gamma}{\lambda_{S R}}\left(\frac{x+1}{y}\right)\right] f_{I_{D}}(x) f_{\gamma_{R D}}(y) d y d x \\
& =\int_{0}^{\infty} f_{I_{D}}(x) \int_{0}^{\infty} \frac{1}{\lambda_{R D}} \exp \left[-\frac{\gamma(x+1)}{\lambda_{S R}} \frac{1}{y}-\frac{y}{\lambda_{R D}}\right] d y d x
\end{aligned}
$$




$$
=2 \sqrt{\frac{\gamma}{\lambda_{S R} \lambda_{R D}}} \int_{0}^{\infty} \sqrt{x+1} K_{1}\left(2 \sqrt{\frac{\gamma}{\lambda_{S R} \lambda_{R D}}} \sqrt{x+1}\right) f_{I_{D}}(x) d x
$$

The first expression is obtained by using the PDF of $\gamma_{R D}$, i.e., $f_{\gamma_{R D}}(y)=\exp \left(-y / \lambda_{R D}\right) / \lambda_{R D}$. Furthermore, (30) is obtained by applying (Eq. 2.3.16.1) in [27]. Accordingly, by substituting (14) into (30), given $\mathbf{X} \triangleq D$ and let $\kappa=\sqrt{x+1}$, (30) becomes:

$$
\begin{aligned}
\mathbb{E}_{I_{D}, \gamma_{R D}}\{\ldots\} & =\sum_{i=1}^{n^{D}} \sum_{j=1}^{m_{i}^{D}} \sum_{n=0}^{j-1}\left(\begin{array}{c}
j-1 \\
n
\end{array}\right) \Phi_{i j}^{D} \frac{v_{[i]}^{-j} \mathrm{e}^{1 / \varphi_{i]}}}{(j-1) !}(-1)^{j-n-1} \\
& \times 4 \sqrt{\frac{\gamma}{\lambda_{S R} \lambda_{R D}}} \int_{1}^{\infty} \kappa^{2 n+2} \exp \left(-\frac{\kappa^{2}}{v_{[i]}}\right) K_{1}\left(2 \sqrt{\frac{\gamma}{\lambda_{S R} \lambda_{R D}}} \kappa\right) d \kappa
\end{aligned}
$$

The above result cannot be expressed in closed-form under our best knowledge. Hence, by substituting (31) and (28) into (26), the desired CDF, $F_{\gamma_{D}}(\gamma)$, is obtained.

\section{References}

[1] H. Gao, W. Ejaz and M. Jo, " Cooperative Wireless Energy Harvesting and Spectrum Sharing in 5G Networks," IEEE Access, Vol.4, pp.3647-3658, July 2017. Article (CrossRef Link).

[2] E. Boshkovska, D. W. K. Ng, N. Zlatanov, A. Koelpin, and R. Schober, "Robust resource allocation for MIMO wireless powered communication networks based on a non-linear EH model,” IEEE Trans. Commun., Vol. 65, No. 5, pp. 1984 - 1999, 2017. Article (CrossRef Link).

[3] J. Zhang, Q. Zhou, D. Ng and M. Jo, "Optimal Energy Efficiency Fairness of Nodes in Wireless Powered Communication Networks,” Sensor, Vol.17, No.9, pp.2125-2146, Sept. 2017.

Article (CrossRef Link).

[4] Dinh-Thuan Do, "Energy-Aware Two-Way Relaying Networks under Imperfect Hardware: Optimal Throughput Design and Analysis," Telecommunication Systems (Springer), Vol. 62, No. 2, pp. 449-459, 2015. Article (CrossRef Link).

[5] Z. Ding and H. V. Poor, "Cooperative energy harvesting networks with spatially random users," IEEE Signal Process. Lett., vol. 20, no. 12, pp. 1211-1214, Dec. 2013. Article (CrossRef Link).

[6] H. Chen, Y. Li, J. L. Rebelatto, B. F. Uchoa-Filho, and B. Vucetic, "Harvest-then-cooperate: Wireless-powered cooperative communications,” IEEE Trans. Signal Process., vol. 63, no. 7, pp. 1700-1711, Apr. 2015. Article (CrossRef Link).

[7] G. Zhu, C. Zhong, H. A. Suraweera, G. K. Karagiannidis, Z. Zhang, and T. A. Tsiftsis, "Wireless information and power transfer in relay systems with multiple antennas and interference," IEEE Trans. Commun., vol. 63, no. 4, pp. 1400-1417, Apr. 2015. Article (CrossRef Link).

[8] C. Huang, R. Zhang, and S. G. Cui, "Throughput maximization for Gaussian relay channel with energy harvesting constraints,” IEEE J. Sel. Areas Commun., vol. 31, no. 8, pp. 1469-1479, Aug. 2013. Article (CrossRef Link).

[9] D. Gunduz and B. Devillers, "Two-hop communication with energy harvesting," in Proc. of 4th IEEE Int. Workshop Comput. Adv. Multi-Sensor Adapt. Process. (CAMSAP), 2011, pp. 201-204. Article (CrossRef Link). 
[10] Minasian, S. ShahbazPanahi, and R. S. Adve, "Energy harvesting cooperative communication systems,” IEEE Trans. Wireless Commun., vol. 13, no. 11, pp. 6118-6131, Nov. 2014, Article (CrossRef Link).

[11] Ahmed, A. Ikhlef, R. Schober, and R. K. Mallik, "Joint power allocation and relay selection in energy harvesting AF relay systems,” IEEE Wireless Commun. Lett., vol. 2, no. 2, pp. 239-242, Apr. 2013. Article (CrossRef Link).

[12] I. Ahmed, A. Ikhlef, R. Schober, and R. K. Mallik, "Power allocation in energy harvesting relay systems," in Proc. of IEEE Veh. Technol. Conf. (VTC), 2012, pp. 1-5. Article (CrossRef Link).

[13] Krikidis, T. Charalambous, and T. S. Thompson, "Stability analysis and power allocation for energy harvesting cooperative networks,” IEEE Signal Process. Lett., vol. 19, no. 1, pp. 20-23, Jan. 2012. Article (CrossRef Link).

[14] Ahmed, A. Ikhlef, R. Schober, and R. K. Mallik, "Power allocation for conventional and buffer-aided link adaptive relaying systems with energy harvesting nodes," IEEE Trans. Wireless Commun., vol. 13, no. 3, pp. 1182-1195, Mar. 2014. Article (CrossRef Link).

[15] S. Guo, C. Wang, and Y. Yang, "Joint mobile data gathering and energy provisioning in wireless rechargeable sensor networks,” IEEE Trans. Mobile Comput., vol. 52, no. 11, pp. 104-110, Dec. 2014. Article (CrossRef Link).

[16] S. Guo, F. Wang, Y. Yang, and B. Xiao, "Energy-efficient cooperative transmission for simultaneous wireless information and power transfer in clustered wireless sensor networks," IEEE Trans. Commun., vol. 63, no. 11, pp. 4405-4417, Nov. 2015, Article (CrossRef Link).

[17] L. R. Varshney, “Transporting information and energy simultaneously,” in Proc. of IEEE Int. Symp. Inf. Theory (ISIT), 2008, pp. 1612-1616. Article (CrossRef Link).

[18] P. Grover and A. Sahai, "Shannon meets Tesla: wireless information and power transfer," in Proc. of IEEE Int. Symp. Inf. Theory (ISIT), 2010, pp. 2363-2367. Article (CrossRef Link).

[19] Y. Chen, D. B. da Costa and H. Ding, "Effect of CCI on WPC With Time-Division Energy and Information Transmission,” IEEE Wireless Commun. Letters, vol. 5, no. 2, pp. 168 - 171, 2016. Article (CrossRef Link).

[20] J. Zhangm X. Yang, Q. Yao, X. Ge, M. Jo and G. Mao, "Cooperative Energy Efficiency Modeling and Performance Analysis in Co-Channel Interference Cellular Networks," The Computer Journal, Vol. 56, No. 8, pp.1010-1019, Aug. 2013. Article (CrossRef Link)

[21] Dinh-Thuan Do, H-S Nguyen, "A Tractable Approach to Analyze the Energy-Aware Two-way Relaying Networks in Presence of Co-channel Interference," EURASIP Journal on Wireless Communications and Networking, vol 271, 2016. Article (CrossRef Link)

[22] Y. Gu, S. Aïssa, "RF-based energy harvesting in decode-and-forward relaying systems: Ergodic and outage capacities," IEEE Trans. Wireless Commun., vol. 14, no. 11, pp. 6425-6434, Nov. 2015. Article (CrossRef Link).

[23] R. Tao, A. Salem, and K. A. Hamdi, “Adaptive relaying protocol for wireless power transfer and information processing,” IEEE Commun. Lett., vol. 20, no. 10, 2016. Article (CrossRef Link).

[24] T.-L. Nguyen and D.-T. Do, "A new look at AF two-way relaying networks: energy harvesting architecture and impact of co-channel interference," Annals of Telecommunications, Vol. 72, No. 11, pp. 669-678, 2017. Article (CrossRef Link).

[25] A. Bletsas, H. Shin, and M. Z. Win, "Cooperative communications with outage-optimal opportunistic relaying,” IEEE Trans. Wireless Commun., vol. 6, no. 9, pp. 3450-3460, Sep. 2007. Article (CrossRef Link).

[26] Jeffrey, Alan, and Daniel Zwillinger, eds. Table of integrals, series, and products. Academic Press, 2007. Article (CrossRef Link).

[27] Prudnikov, A. P., Brychkov, Y. A. \& Marichev, O. I. (1 \& 2) 1986. Integrals and Series. New York: Gordon and Breach Science Publishers. 


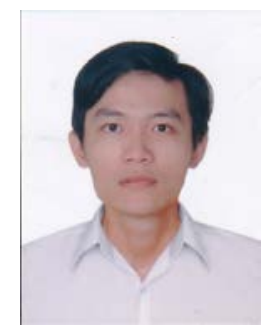

Thanh-Luan Nguyen received B.S. degree in Electronics and Telecommunications Engineering from Ho Chi Minh University of Technology and Education in 2016 with First Honor Graduate. He is currently pursuing his MS. degree in Telecommunication Engineering at Bach Khoa University, Ho Chi Minh, Viet Nam. His major interests include cooperative (relay) communications, energy harvesting and non-orthogonal multiple access

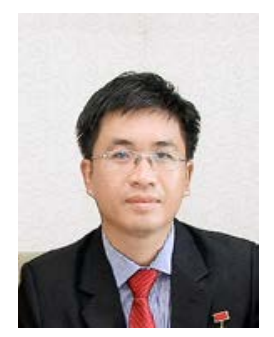

Dinh-Thuan Do received the B.S. degree, M. Eng. degree, and Ph.D. degree from Vietnam National University (VNU-HCMC) in 2003, 2007, and 2013 respectively, all in Communications Engineering. He was a visiting Ph.D. student with Communications Engineering Institute, National Tsing Hua University, Taiwan from 2009 to 2010. Prior to joining Ton Duc Thang University, he was senior engineer at the VinaPhone Mobile Network from 2003 to 2009. Dr. Thuan was the recipient of the 2015 Golden Globe Award by Ministry of Science and Technology. He is currently Assistant Professor at the Wireless Communications \& Signal Processing Lab (WICOM LAB). His research interest includes signal processing in wireless communications network, mmWave, device-to-device networks, non-orthogonal multiple access, full-duplex transmission and energy harvesting. 